\title{
Osteotomias femorais distais com cunha de fechamento medial-estudo retrospectivo*
}

\section{Closing-wedge Distal Femoral Osteotomy-Retrospective Study}

\author{
Pedro Barreira Cabral ${ }^{1}$ Diego Costa Astur ${ }^{2}$ Eduardo Vasconcelos Freitas ${ }^{1}$ Bruno Silveira Pavei ${ }^{1}$ \\ Camila Cohen Kaleka ${ }^{1}$ Moises Cohen ${ }^{2}$
}

${ }^{1}$ Instituto Cohen São Paulo, São Paulo, SP, Brasil

2 Departamento de Ortopedia e Traumatologia, Escola Paulista de Medicina, Universidade Federal de São Paulo, São Paulo, SP, Brasil
Address for correspondence Pedro Barreira Cabral, Instituto Cohen São Paulo, São Paulo, SP, Brasil (e-mail: pedro.barreira.c@gmail.com).

Rev Bras Ortop 2019;54:198-201.

\section{Resumo \\ Palavras-chave \\ - osteotomia \\ - fêmur \\ - joelho \\ - osteoartrite \\ - geno valgo}

\section{Abstract}

Objetivo Descrever a técnica cirúrgica da osteotomia femoral com cunha de fechamento medial e uma série de casos submetidos a essa técnica.

Métodos Foram avaliados 26 pacientes submetidos a osteotomia femoral distal com cunha de fechamento medial de 2002 a 2013. Os prontuários e exames de imagem de todos os pacientes foram revisados para avaliação do grau de correção e estado atual. Resultados Dos 26 pacientes operados, 12 eram do sexo masculino e 14 do feminino. A idade média foi de 47,15 anos. Em todos os casos, obteve-se alinhamento neutro em relação ao eixo anatômico. A maioria dos pacientes alcançou a consolidação óssea da osteotomia com seis semanas. Não foram observados casos de sangramentos durante a cirurgia. Um paciente apresentou retardo da consolidação óssea. Um paciente apresentou desconforto sobre a placa, foi necessária sua retirada. Um paciente apresentou infecção superficial sem necessidade de revisão da osteotomia. Não foram observados casos de trombose venosa profunda e tromboembolismo pulmonar. Até o momento não houve conversão para artroplastia total de joelho.

Conclusão $\mathrm{O}$ tratamento com osteotomia femoral distal com cunha de fechamento medial manteve a correção proposta em pacientes com seguimento de até 15 anos.

Objective To describe the surgical technique of distal closing-wedge femoral osteotomy and a cases series submitted to this technique.

Methods A total of 26 patients submitted to medial closing-wedge distal femoral osteotomy from 2002 to 2013 were evaluated. All of the patients had their medical files and imaging exams reviewed to evaluate the degree of correction and their current state.
Trabalho desenvolvido no Centro de Traumatologia do Esporte, Departamento de Ortopedia e Traumatologia, Escola Paulista de Medicina, Universidade Federal de São Paulo, São Paulo, SP, Brasil. Publicado originalmente por Elsevier Ltda. received

July 24,2017

accepted

October 24, 2017
DOI https://doi.org/

10.1016/j.rbo.2017.10.007. ISSN 0102-3616.
Copyright $\odot 2019$ by Sociedade Brasileira License terms de Ortopedia e Traumatologia. Published by Thieme Revnter Publicações Ltda, Rio de Janeiro, Brazil 


\section{Keywords \\ - osteotomy \\ - femur \\ - knee \\ - osteoarthritis \\ - genu valgum}

Results Out of the 26 patients, 12 were male and 14 were female. Their mean age was 47.15 years old. In all of the cases, a neutral alignment related to the anatomical axis was achieved. Most of the patients presented bone healing at 6 weeks. There were no cases of bleeding during the surgery. One patient presented with delayed bone healing. One patient complained of plaque-related discomfort, requiring the removal of the device. One patient had a superficial infection, but no osteotomy revision was needed. There were no cases of deep venous thrombosis or of pulmonary thromboembolism. To date, there has been no conversion to total knee replacement.

Conclusion Treatment with medial closing-wedge distal femoral osteotomy sustained the proposed correction in patients with up to 15 years of follow-up.

\section{Introdução}

A osteoartrite (OA) é uma doença degenerativa das articulações que afeta a população em geral. O joelho é a principal articulação periférica acometida, resultando em uma progressiva perda de função, dor e rigidez articular. É estimado que cerca de um décimo da população com mais de 50 anos será afetada por essa patologia. ${ }^{1}$

A técnica cirúrgica da osteotomia dos ossos longos dos membros inferiores (fêmur e tíbia) tem como objetivo a mudança do alinhamento do membro afetado, desacelera o processo evolutivo da OA. Com isso, causa melhora da dor, da função do membro e pode postergar os procedimentos substitutivos da articulação, como a artroplastia de joelho em até 10 anos. $^{2}$

A osteotomia femoral distal é um procedimento bem aceito para o tratamento de OA unicompartimental em estágios iniciais decorrentes do joelho em valgo. Chahla et al citam que os melhores resultados são obtidos quando as osteotomias são feitas em pacientes com idade inferior a 55 anos. ${ }^{1,3-5}$

A osteotomia femoral pode ser de abertura lateral ou fechamento medial. Em média, deve-se corrigir a deformidade em busca do alinhamento anatômico neutro. Entretanto, não existem evidências na literatura sobre a superioridade da técnica com cunha de fechamento medial em relação a cunha de abertura lateral. ${ }^{4,6}$

O objetivo do estudo é descrever a técnica cirúrgica e uma série de casos submetidos a osteotomia femoral com cunha de fechamento medial em um serviço de referência e avaliar as características atuais decorrentes desse procedimento em seguimento de até 15 anos.

\section{Materiais e métodos}

Este estudo foi avaliado e aprovado pela Comissão de Ética e pesquisa dessa instituição sob o número CAAE 01609812.9. 0000.5505 .

Foram avaliados 26 pacientes submetidos a osteotomia femoral distal com cunha de fechamento medial de 2002 a 2013. Um dos pacientes foi submetido a osteotomia bilateral, total de 27 joelhos operados.

Todos os pacientes tiveram seus prontuários e exames de imagem revisados, foram coletados dados referentes a gênero, idade no ato cirúrgico, lateralidade, deformidade, complicações intraoperatórias. Em seguida, foram analisados clinicamente e com exame de imagem para caracterizar o estado atual dos pacientes: falha no tratamento, necessidade de conversão para artroplastia total de joelho, bem como outras complicações.

\section{Avaliação inicial}

O principal indicador de correção do membro inferior acometido foi a deformidade em valgo maior do que $12 \circ$. Para o planejamento pré-operatório foram solicitadas radiografias em anteroposterior e em perfil do lado a ser operado e uma radiografia panorâmica dos membros inferiores para cálculo do valgo e determinação se a deformidade era no fêmur distal. Os critérios para correção foram: os pacientes não podiam apresentar dor difusa ou inespecífica no joelho ou queixa principal de dor na articulação patelofemoral, ter sido submetidos a meniscectomia prévias no compartimento destinado à sustentação do peso, apresentar artrose no compartimento destinado à sustentação do peso, ter o diagnóstico subjacente de doença inflamatória e necessitavam arco de movimento de pelo menos 90 graus e contratura em flexão menor do que 10 graus.

\section{Técnica cirúrgica}

Com o paciente posicionado em decúbito dorsal sobre uma mesa radiotransparente, identificamos a superfície articular por meio de palpação e radioscopia. Fizemos uma incisão de aproximadamente $10 \mathrm{~cm}$, que se estendia a partir da linha articular medial do joelho em sentido proximal. Em seguida, fizemos a dissecção romba do vasto medial para expor o côndilo e o córtex femoral medial. Com isso, nenhuma estrutura neurovascular foi exposta ou colocada em risco durante o acesso cirúrgico, o que tornou segura a exposição da superfície óssea necessária para a osteotomia. Fizemos a marcação da linha médio diafisária com um bisturi elétrico e com uma caneta de Codman para evitar desvio angulares no momento da estabilização com a placa. Com o uso da chapaguia, posicionamos pinos paralelos para auxílio do corte da cunha. Em seguida, fizemos o corte da parte proximal da osteotomia. Colocamos o guia de corte cunha e fios com o corte angular pré-definido para cada caso e fizemos o corte e a retirada de aproximadamente $75 \%$ da cunha, considerada 

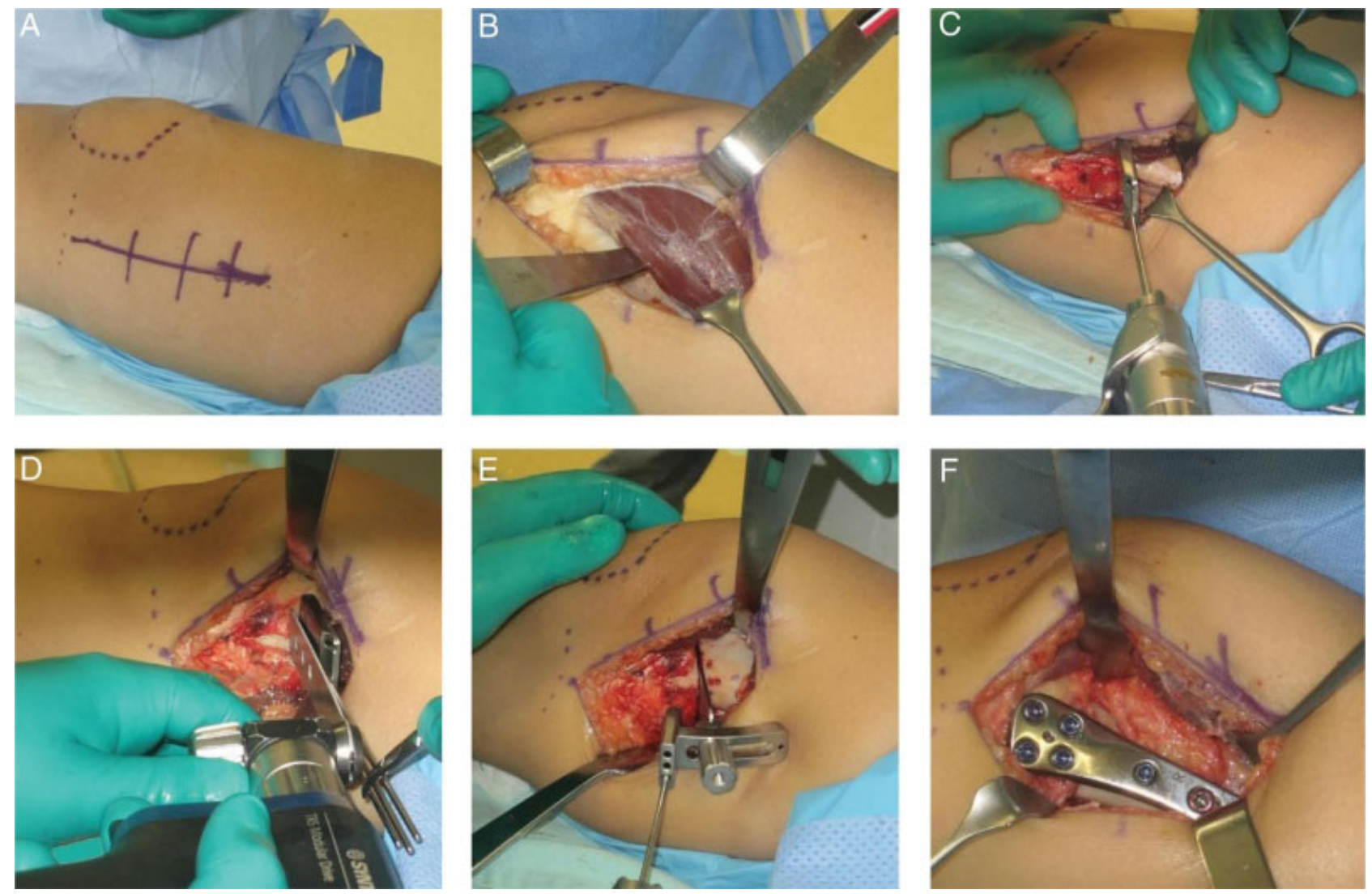

Fig. 1 A, demarcação da linha articular, patela e do acesso cirúrgico; B, colocação de afastador subvasto; C, colocação de pinos paralelos com chapa guia; D, feitura da parte proximal da osteotomia; E, colocação de pinos no guia de corte cunha para finalização da osteotomia; F, placa posta após osteotomia.

parcial. Checamos com radioscopia e fizemos a estabilização da osteotomia com placa lâmina angulada em 90o, nos casos mais antigos e bloqueada nos mais recentes, com parafusos proximais e parafusos distais a osteotomia (- Fig. 1).

\section{Resultados}

Dos 26 pacientes operados, 12 eram do sexo masculino e 14 do feminino. A idade média foi de 47,15 anos (mínimo de 25 e máximo de 61). Com relação ao lado, 13 pacientes foram submetidos a osteotomias do fêmur esquerdo, 12 do lado direito e um paciente foi submetido a osteotomia bilateral, total de 27 osteotomias femorais distais.

A deformidade em valgo variava entre 13 e 18 graus antes da cirurgia. Após a osteotomia, foi obtido em todos os casos o alinhamento anatômico em neutro, variou de 0 a 1 grau de valgo.

Durante o ato cirúrgico, não tivemos sangramento relevante. Também não foi relatada lesão neurovascular devido ao acesso cirúrgico.

A maioria dos pacientes obteve a consolidação óssea da osteotomia com seis semanas (mínimo de seis e máximo de 16). A consolidação foi definida de acordo com acompanhamento radiográfico seriado.

Uma paciente apresentou retardo na consolidação, evoluiu com fratura após queda de altura. Foi submetida a estabilização medial e lateral para consolidação óssea.
Um paciente apresentou desconforto sobre a placa persistente ao tratamento conservador. Após um ano optou-se optado pela retirada do material de síntese. Depois desse procedimento cirúrgico, o paciente melhorou das queixas apresentadas.

Um paciente teve infecção superficial, foi submetido a limpeza cirúrgica e antibioticoterapia, sem necessidade da retirada da placa e dos parafusos.

Não houve casos de trombose venosa profunda e tromboembolismo pulmonar. Nenhum paciente teve conversão para artrosplastia de joelho até o momento em um acompanhamento de pelo menos cinco anos.

\section{Discussão}

A osteotomia femoral varizante é uma técnica cirúrgica usualmente usada para correção de deformidade em valgo do joelho. Na presença de uma sobrecarga do compartimento lateral do joelho, ela pode ser feita em pacientes selecionados para diminuir a pressão aumentada entre o côndilo femoral lateral e o planalto tibial lateral. Em 27 osteotomias feitas, a idade média foi de 47,15 anos, de 25 a 61 . 0 paciente mais jovem a fazer osteotomia ( 25 anos) era jogador de futebol, já havia feito meniscectomia lateral prévia e evoluiu com quadro de osteoartrite que progrediu rapidamente, provavelmente devido a alta demanda esportiva. ${ }^{5,6}$ Chahla et al, ${ }^{4}$ em uma revisão sistemática com 248 joelhos, obtiveram a idade média de 48,9 anos. 
Apesar de OA ser mais prevalente no sexo feminino, ${ }^{6}$ a literatura não é conclusiva sobre em qual sexo o procedimento é mais feito. ${ }^{6,7}$ Em nosso estudo, 12 pacientes submetidos a osteotomia eram do sexo masculino e 14 do feminino.

A principal discussão sobre a osteotomia femoral varizante distal do fêmur segue sobre qual técnica deve ser aplicada: cunha de fechamento medial ou cunha de abertura lateral. A técnica que usa a cunha de abertura lateral é mais popular. Provavelmente isso ocorre pois grande parte dos cirurgiões que a aplicam a considera mais fácil e mais segura, devido à familiaridade com o acesso cirúrgico. ${ }^{4,8}$ As osteotomias com cunha de fechamento medial pareciam ter mais complicações, muitas dessas informações vieram de estudos mais antigos, nos quais os cirurgiões usavam como métodos de fixação grampos em vez de mais placas e parafusos. ${ }^{9}$ Acreditamos que a técnica com cunha de fechamento medial permite que a correção seja mais anatômica e com tempo de consolidação menor. Além disso, dispensa a necessidade de enxerto ósseo e carga sobre o membro operado mais precoce. O risco neurovascular, comumente descrito como principal causa da não popularização da técnica, é baixo quando a via cirúrgica é adequadamente feita. Na nossa série, não houve caso de complicação ou acometimento neurovascular., ${ }^{4,8}$ Visser et $\mathrm{al}^{8}{ }^{8}$ em um estudo cadavérico, corroboram esse dado e falam da segurança do acesso medial para a colocação da placa. Eles citam ainda que o dano ao vasto medial e o risco de lesões neurovasculares é mínimo, mesmo com a técnica minimamente invasiva.

A maioria dos pacientes obteve consolidação óssea com seis semanas após a cirurgia. Em média, os estudos citam que a consolidação óssea ocorra entre seis e oito semanas. Apenas um paciente apresentou retardo na consolidação. Paciente esse que era fumante de longa data e optou por não parar de fumar durante o pós-operatório, o que corrobora o risco aumento de retardo na consolidação óssea. ${ }^{4,10,11}$

Além disso, o desconforto sobre a placa é uma complicação relatada na literatura. ${ }^{4} \mathrm{Um}$ de nossos pacientes apresentou essa complicação. Um dos motivos prováveis era o paciente apresentar baixo peso e a massa muscular do vasto medial não ser capaz de cobrir bem a placa, o que gerava tal desconforto., Forkel et al, em um acompanhamento de 23 pacientes submetidos a osteotomia femoral com cunha de fechamento medial, mostraram o desconforto na placa em 16 pacientes. Optamos pela retida do material de síntese após tempo adequado. 0 paciente teve melhoria dos sintomas após a retirada. ${ }^{12,13}$

Por fim, a infecção cirúrgica é um evento que, apesar de medidas tomadas pré-operatórias, intraoperatórias e pósoperatórias, em alguns casos pode ocorrer. Em um paciente deste estudo foi relatada infecção superficial, tratada com antibioticoterapia e limpeza cirúrgica. Não foi necessária revisão do material de síntese. ${ }^{4,9-12}$

Usamos em nossos pacientes, sempre que necessário, medidas farmacológicas e não farmacológicas para evitar um dos eventos mais graves relatados na literatura que é a trombose venosa profunda e a embolia pulmonar. Contudo, não tivemos pacientes com essa complicação. ${ }^{2,4}$

Wylie et $\mathrm{al}^{10}{ }^{10}$ em outra revisão sistemática, mostraram uma taxa de sobrevivência das osteotomias femorais com cunha de fechamento medial em 10 anos de $82 \%$. Chahla et $\mathrm{al}^{4}$ mostram que a taxa de sobrevivência média variou de $64 \%$ a $89,9 \%$ no mesmo seguimento de 10 anos. Quando o seguimento foi de até 15 anos, esse mesmo estudo mostrou uma taxa de sobrevivência bem menor, de $45 \%$ a $78,9 \%$. No nosso seguimento de até 15 anos não foi necessária a conversão para artroplastia do joelho até o momento., ${ }^{4,10}$

\section{Conclusão}

A osteotomia femoral distal com cunha de fechamento medial é um procedimento que manteve a correção proposta em pacientes com seguimento de até 15 anos, evoluiu em pouquíssimos casos com complicações decorrentes da cirurgia.

Conflitos de interesse

Os autores declaram não haver conflitos de interesse.

\section{Referências}

1 Hussain SM, Neilly DW, Baliga S, Patil S, Meek R. Knee osteoarthritis: a review of management options. Scott Med J 2016;61(01):7-16

2 Brouwer RW, Raaij van TM, Bierma-Zeinstra SM, Verhagen AP, Jakma TS, Verhaar JA. Osteotomy for treating knee osteoarthritis. Cochrane Database Syst Rev 2007;(03):CD004019

3 Haviv B, Bronak S, Thein R, Thein R. The results of corrective osteotomy for valgus arthritic knees. Knee Surg Sports Traumatol Arthrosc 2013;21(01):49-56

4 Chahla J, Mitchell JJ, Liechti DJ, Moatshe G, Menge TJ, Dean CS, et al. Opening- and closing-wedge distal femoral osteotomy: a systematic review of outcomes for isolated lateral compartment osteoarthritis. Orthop J Sports Med 2016;4(06):2325967116649901

5 Hoorntje A, Witjes S, Kuijer PPFM, Koenraadt KLM, van Geenen RCI, Daams JG, et al. High rates of return to sports activities and work after osteotomies around the knee: a systematic review and meta-analysis. Sports Med 2017;47(11):2219-2244

6 Leone JM, Hansse AD. Osteotomia ao redor do joelho: perspectiva americana. In: Scott NW, ed. Insall \& Scott cirurgia do joelho. 5 a. ed. Rio de Janeiro: Elsevier; 2014:2070-2105

7 Murphy GA. Total ankle arthroplasty. In: Azar FM, Beaty JH, Canale ST, eds. Campbell's operative orthopaedics. Philadelphia: Elsevier; 2017:508-534

8 Visser J, Brinkman JM, Bleys RL, Castelein RM, van Heerwaarden RJ. The safety and feasibility of a less invasive distal femur closing wedge osteotomy technique: a cadaveric dissection study of the medial aspect of the distal femur. Knee Surg Sports Traumatol Arthrosc 2013;21(01):220-227

9 Gardiner A, Richmond JC. Periarticular osteotomies for degenerative joint disease of the knee. Sports Med Arthrosc Rev 2013;21 (01):38-46

10 Wylie JD, Jones DL, Hartley MK, Kapron AL, Krych AJ, Aoki SK, et al. Distal femoral osteotomy for the valgus knee: medial closing wedge versus lateral opening wedge: a systematic review. Arthroscopy 2016;32(10):2141-2147

11 Lobenhoffer P, Kley K, Freiling D, van Heerwaarden R. [Medial closed wedge osteotomy of the distal femur in biplanar technique and a specific plate fixator]. Oper Orthop Traumatol 2017;29(04):306-309

12 Shantz JS, Marcucio R, Kim HT, Miclau T. Bone and cartilage healing. In: Court-Brown CM, Heckman JD, Mcqueen MM, Ricci WM, Tornetta P, eds. Rockwood and Green's fractures in adults. Philadelphia: Wolters Kluwer; 2015:109-125

13 Forkel P, Achtnich A, Metzlaff S, Zantop T, Petersen W. Midterm results following medial closed wedge distal femoral osteotomy stabilized with a locking internal fixation device. Knee Surg Sports Traumatol Arthrosc 2015;23(07):2061-2067 